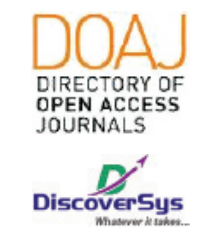

Published by DiscoverSys

\section{Positive nikolsky sign and "pinpoint lake of pus" in acute generalized exanthematous pustulosis: A case report}

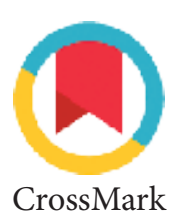

Ketut Alit Pinidha Savitri, ${ }^{1 *}$ Ketut Suteja Wibawa ${ }^{2}$ Komang Harry Supradnyan $^{2}$
${ }^{1}$ General Practitioner, Singaraja Regional Hospital, Buleleng, BaliIndonesia

${ }^{2}$ Dermatology and Venereology Department, Singaraja Regional Hospital, Buleleng, Bali-Indonesia
*Correspondece to:

Ketut Alit Pinidha Savitri, General Practitioner, Singaraja Regional Hospital, Buleleng, Bali-Indonesia nidhasavitri@yahoo.com

Received: 2019-08-03 Accepted: 2020-01-03

Published: 2020-03-03

\title{
ABSTRACT
}

Introduction: Acute generalized exanthematous pustulosis (AGEP) is a severe, usually drug-related reaction, characterized by an acute onset of mainly small non-follicular pustules on an erythematous base and spontaneous resolution usually within two weeks. Current report aims to present management of AGEP in our center at Singaraja Regional Hospital, Bali-Indonesia.

Case: A 78 years old woman with a history of acute coronary syndrome, was reported to have a severe allergic drug reaction. Patients reported taking cefixime medication for the past 2 days because of acute bronchitis. The patient was hospitalized because of the complaint, and diffuse miliary pustules were found above the base of erythematous skin. On examination of lesions, the Nikolsky sign was positive on lesions in the back and abdomen without any tenderness. Histopathological examination was suitable for the diagnosis of acute generalized exanthema pustulosis (AGEP). Systemic intravenous corticosteroid with oral antihistamines was choosen as a primary treatment for this patient.

Conclusion: Treatment is based on the removal of the causative drug, supportive care, infection prevention and use of potent topical or systemic steroids

Keywords: acute generalized exanthema pustulosis, drug rush, lake of pus, beta-lactam, cefixime

Cite this Article: Savitri, K.A.P., Wibawa, K.S., Supradnyan, K.H. 2020. Positive nikolsky sign and "pinpoint lake of pus" in acute generalized exanthematous pustulosis: A case report. Intisari Sains Medis 11(1): 97-99. D0I: 10.15562/ism.v11i1.579

\section{INTRODUCTION}

Acute generalized exanthema pustulosis (AGEP) is a severe cutaneous reaction caused by a drug allergy. The clinical manifestation of AGEP is a nonfollicular miliary pustule with erythematous base. Lesions can be a picture of the target lesion. This can be attributed to the risk of death by $2 \%$ which is compared to TEN mortality rate of $25-30 \%$. On complete blood tests can be found an increase in leukocytes, neutrophils and eosinophilia. Skin reactions can appear 24-48 hours after drug exposure, depending on how infectious the exposure agent is. A number of drugs, especially cephalosporin and macrolide antibiotics are associated as the etiology. Besides anticonvulsant drugs, NSAIDs, simvastatin, nifedipine, herbal medicines and insect bites have been reported as triggers of AGEP.

\section{CASE}

A woman, 78 years old with a history of coronary heart disease for the past 2 years and routinely took furosemide, bisoprolol, and valsartan. One week before the rash appeared, patient complained of coughing and seek for the treatment to pulmonary doctor, the X-ray results show the patient has acute bronchitis. After 5 days hospitalization, the patient returned home with improved condition and brought cefixime, salbutamol, and paracetamol. Two days later after taking the drug, the patient had burning sensation on the skin accompanied by itching. One day later a reddish rash appeared and pustules arise in thighs and neck fold which quickly spread throughout the body. Examination on ER revealed diffuse erythematous-based papules on nearly $90 \%$ of the body area (except for a small area of the face, lips and genitals). In the lesions in the arm and abdomen there are pinpoint pustules which give an appearance of 'lake of pus'. Evaluation of lesions showed a positive nikolsky sign on the back, abdomen, and right upper thigh areas. There are no lesions in the mucosa area such as oral, nasal, ocular and genital mucosa. There were no purpoid atypical lesion and hemorrhagic bullae. Present status tests revealed awareness of compost mentis, normal blood pressure, axillary temperature $38,7^{\circ} \mathrm{C}$. Blood tests obtained $60.6 \mathrm{k} / \mathrm{uL}$ leukocytes, $54.3 \%$ neutrophils and $1.79 \%$ lymphocytes. The kidney function test found an increase in creatinine $1.89 \mathrm{mg} / \mathrm{dl}$ and urea 113.0. Examination of electrolytes was obtained potassium $3.3 \mathrm{mmol} / \mathrm{L}$. Liver function and blood glucose are obtained normal. Patients were consulted to cardiology with ACS NSTEMI and carried out joint treatment in the ICCU room. Histopathological result taken from the right upper extremity lesions were obtained by subcorneum blister, in which there were inflammatory cells of 


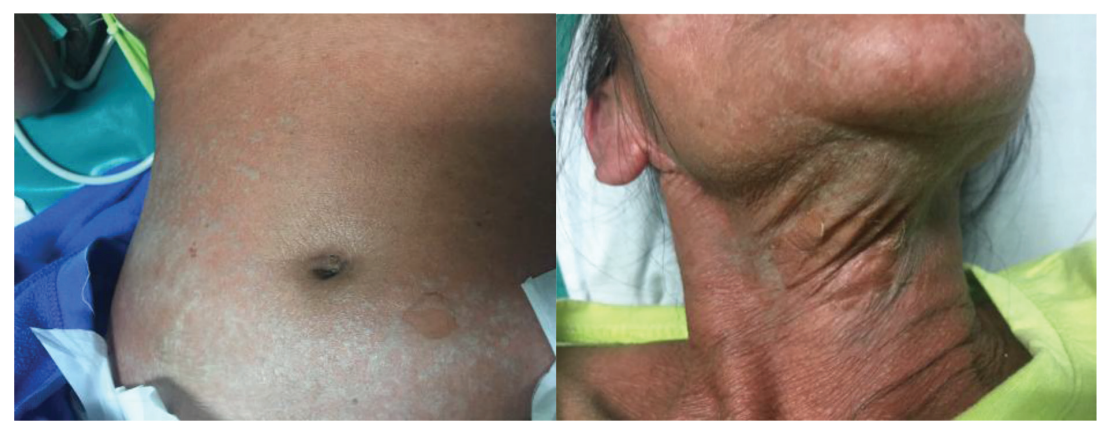

Figure 1 Clinical manifestations show papules with erythematous base forming a "lake of pus" and a positive Nikolsky sign.

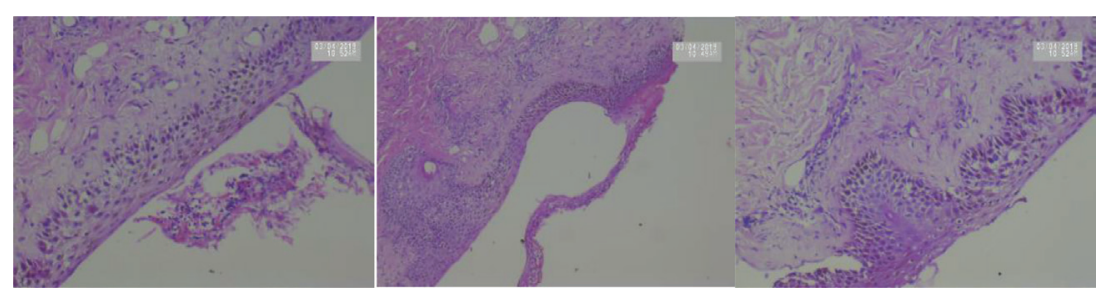

Figure 2 AGEP eruption on the skin: a histopathology with $100 \times$ magnification shows bullous sub-corneal in the epidermal layer surrounded by inflammatory cells PMN and neutrophils. In the dermis layer obtained PMN inflammatory cells, neutrophils, lymphocytes and a little erythrocytes

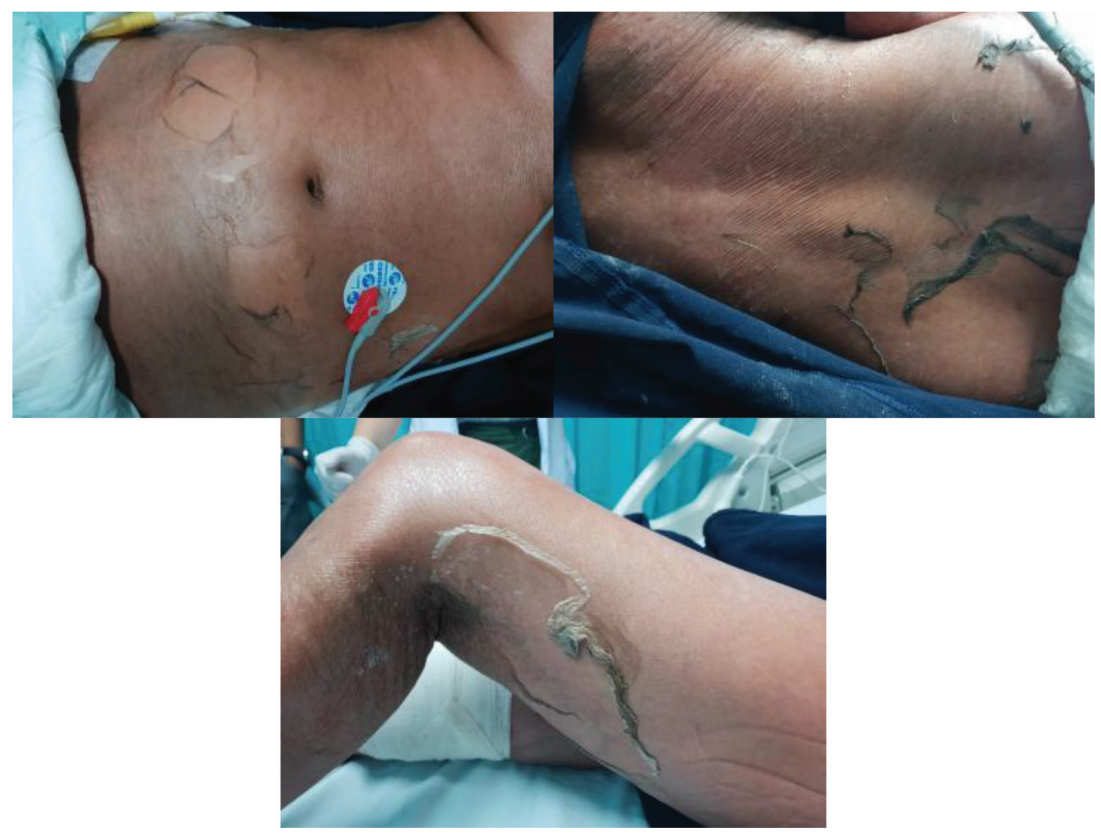

Figure 3 All lesions are desquamated after the sixth-day treatment

PMN and neutrophils. Using the EuroSCAR criterion, the diagnosis of AGEP made by the suspicious drug is cefixime. Suspicious drug stopped, followed by administration of $62.5 \mathrm{mg} /$ day intravenous methylprednisolone with oral antihistamines and topical desoximetasone showed gradual improvement of the lesions in less than 7 days. On the third day treatment, the nicholsky was negative and no new pustules appeared. On the sixth day of treatment almost all lesions have undergone desquamation. The dose of methylprednisolone continued to be reduced within 7 days of treatment according to repair of the lesion. Laboratory tests afterward showed a significant improvement, namely a decrease in leukocyte levels to $9.58 \mathrm{k} / \mathrm{uL}$ with $7.37 \%$ neutrophils and $0.66 \%$ lymphocytes. Kidney function found a decrease in creatinine $0.83 \mathrm{mg} / \mathrm{dL}$ and urea 37.6. Electrolyte examination was obtained potassium $4,1 \mathrm{mmol} / \mathrm{L}$.

\section{DISCUSSION}

Acute Generalized Exanthema Pustulosis (AGEP) is a severe cutaneous reaction caused by a drug allergy. Generally AGEP appears as a nonfollicular pustule above the base of erythematous skin. This can be attributed to the $2 \%$ mortality rate compared to TEN which reaches $25-30 \%$. Increased leukocytes are always found in AGEP patients, followed by an increase in neutrophils and accompanied by fever. Skin lesions usually appear 24-48 hours or can reach less than 10 days after exposure to drugs or infectious agents followed by rapid desquamation within a few days. Cefixime is a beta-lactam class antibiotic which the literature has been mentioned as one of the causes of AGEP. Complications in AGEP are very rare but impaired renal function has been reported to occur in AGEP patients. Positive nikolsky sign in AGEP patients can occur but is not a specific matter because it is more directed to the pseudo-Nikolsky sign. Patients reported having similar lesions with SJS/TEN. Both AGEP and SIS/TEN are severe cutaneous reactions and their pathogenesis cannot be clearly understood but generally triggered by drugs, SJS/TEN is considered a series of diseases characterized by keratinocyte necrosis, secondary detachment of the epidermis, and mucous membrane involvement. Even so, AGEP is considered a milder condition with less involvement and with a shorter latency and faster resolution after the withdrawal of the culprit drug. Confirmation with histopathological findings was obtained in the form of sub-corneal in the surrounding epidermal layer obtained by inflammatory cells PMN and neutrophils. AGEP scoring using Eurroscar has become a reference for diagnosis, the total score between $8-12$ is mentioned as definitive diagnosis. In the case of a score of 11, the diagnosis of AGEP can be established.

\section{CONCLUSION}

An AGEP case that is thought to have been caused by beta-lactam group of antibiotics (cefixime) in a patient with acute coronary syndrome has been reported. The diagnosis of AGEP in cases is 
based on 5 specific criteria, namely small $(<5 \mathrm{~mm})$ subcorneal or intraepidermal pustules above erythema skin, large numbers, typical histopathological changes, fever, increased leukocytes, and repair of lesions less than 7 days, total score using euroSCAR is 11 and the results of histopathological examination are in the form of subcorneal pustules. Management in this case is to stop taking the suspicious drug and administering $62.5 \mathrm{mg}$ intravenous methylprednisolone and tap-off according to the recovery of the lesion. Also, oral antihistamine and desoximetasone topical added. Within 7 days after the therapy, clinical and laboratory improvements were found. The patient's prognosis in the case is dubious ad bonam.

\section{ETHICAL CONSIDERATION}

Patient has received a signed inform consent regarding publication of their respective photograph in journal article.

\section{REFERENCE}

1. Christina Y, Wong M, Steven D, Billings M, Christine W. Positif Nikolsky Sign and Pinpoint Lake of Pus. The National Society for Cutaneous Medicine. 2017; 1(2):86-9.
2. Gaud C, Florence A, Christian C, Tristan F. Acute Generalised Exanthematous Pustulosis due to Prisyinamycin. BMJ Case Report. 2013; 10(11): 1-4.

3. Michael M, Nektaria S, Fani G. Acute Generalized Exanthematous Pustulosis (AGEP) Triggered by a Spider Bite. Journal of Allergology International. 2009; 58(2): 301-03.

4. Kumutnart H. Five Year Retrospective Review of Acute Generalized Exanthema Pustulosis. Dermatology Research and Practice. 2015;8(1): 1-6.

5. Mohammed H, Salhamooud A, Abdel G, Nabeel N. Acute Generalized Exanthematous Pustulosis: An Unusual Side Effect of Meropenem. Indian Journal Dermatology. 2010; 55(2): 176-77.

6. Siew C, Yi S, Nai L, Evelyn S, Xiao Y, Nalini N, Madhavan. Clinical Characteristics, Culprit Drugs and Outcome of Patients with Acute Generalised Exanthematous Pustulosis seen in Hospital Sultanah Aminah, Johor Bahru. Medical Journal Malaysia. 2018; 73(4): 220-24.

7. Mindy M, Sampson, Olga K, Julie V, Beata C. A Plethora of Pustules: Acute Generalized Exanthematous Pustulosis. The American Journal of Medicine. 2018; 131(6): 639-41.

8. Oscar M, Rosario C, Elena S, Luis B. Acute Generalized Exanthematous Pustulosis Simulating Stevens-Johnson Syndrome/ Toxic Epidermal Necrolysis Associated with use of Vismodegib. JAAD Case Report. 2018; 4(1): 123-25.

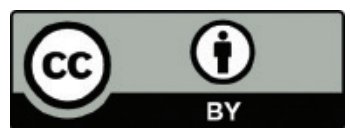

This work is licensed under a Creative Commons Attribution 